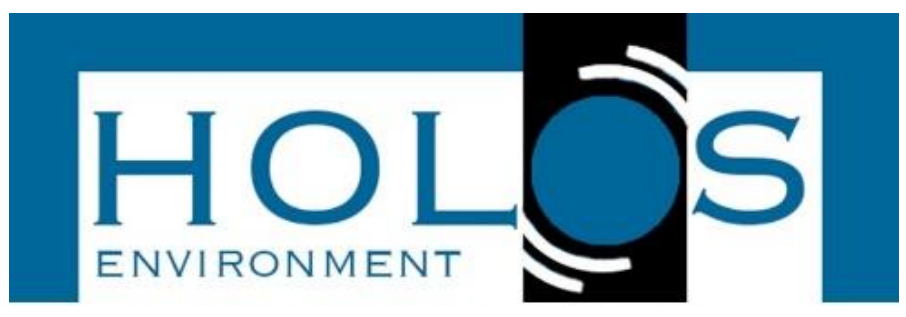

\title{
ESTUDO DA CAPACIDADE DE REMOÇÃO DE AZUL DE METILENO PELA BIOMASSA DA CASCA DO LIMÃO TAITI (Citus Latifolia)
}

\section{STUDY OF METHYLENE BLUE REMOVAL CAPACITY BY TAITI LEMON SHELL BIOMASS (Citus Latifolia)}

\author{
Djeson Mateus Alves da Costaํ'; José Jackson Soares de Melo
}

Artigo recebido em: 12/04/2018 e aceito para publicação em: 12/12/2018. DOI: http://dx.doi.org/10.14295/holos.v18i2.12285

Resumo: Foi realizado um estudo para verificar o $\mathrm{pH}$ em que a carga na superfície dos resíduos da casca do limão taiti era nula ( $(\mathrm{H}$ PCz) e a capacidade de adsorção de azul de metileno, existente em sistemas aquosos artificiais, por essa biomassa, lavada com água destilada e modificada quimicamente com $\mathrm{NaOH} 0,10 \mathrm{M}$. Os ensaios foram realizados em triplicata, utilizando sistema em batelada e as determinações dos teores do azul de metileno, adsorvido pelas fibras, por espectrofotometria em UVVis. Os pontos de carga zero ( $\mathrm{pH}$ PCZ) das duas biomassas não diferiram estatisticamente, apresentando valor médio igual a 5,72. A taxa de adsorção máxima foi igual a 99,58\% $(49,691 \mathrm{mg} / \mathrm{g})$ na biomassa modificada, 17,79\% superior que na biomassa lavada. A adsorção do azul de metileno, pelas fibras estudadas, ajustou-se ao modelo da isoterma de Freundlich.

Palavras-chave: Adsorção. Limão taiti. Ponto de Carga Zero. Isoterma.

Abstract: A study was carried out to verify the $\mathrm{pH}$ at which the surface charge of the residues of the taiti lemon peel was zero ( $\mathrm{pH}$ PCz) and, in artificial aqueous systems, the adsorption capacity of methylene blue by this biomass, washed with distilled water and chemically modified with $\mathrm{NaOH} 0,10 \mathrm{M}$. The experiments were carried out in triplicate, using a batch system and the methylene blue contents, adsorbed by the fibers, by UV-Vis spectrophotometry determinations. The zero load points ( $\mathrm{pH} P \mathrm{PCZ}$ ) of the two biomasses no differ statistically, presenting an average value of 5.72. The maximum adsorption rate was $99.58 \%$ (49.691 $\mathrm{mg} / \mathrm{g}$ ) in the modified biomass, $17.79 \%$ higher than in the washed biomass. The adsorption of methylene blue by the studied fibers was adjusted to the Freundlich isotherm model.

Palavras-chave: Adsorption. Taiti lemon. Point of zero charge. Isotherm.

\section{INTRODUÇÃO}

A contaminação do meio ambiente, em especial dos aportes hídricos, é um grande problema que tem gerado muita preocupação para a sociedade contemporâ-

\footnotetext{
${ }^{1}$ Instituto Federal de Educação, Ciência e Tecnologia do Rio Grande do Norte. E-mails: (djeson.mateus@ifrn.edu.br, jacksonmelo1855@gmail.com)
} 
nea. Em geral, essa poluição é uma consequência do crescimento populacional e do aumento das atividades industriais e agrícolas.

As atividades industriais, principalmente do ramo têxtil, com o uso de uma grande variedade de corantes, geram grandes volumes de cargas orgânicas que são descartadas no ambiente, juntamente com outros efluentes, potencializando alto risco de contaminação do solo e das águas existentes nos reservatórios naturais (RODRIGUES et al., 2010).

Estimativas indicam que em 2003 o setor têxtil produziu mais de 700 mil toneladas dos mais variados tipos de corantes, das quais $30 \%$ se perderam nos resíduos durante as etapas secundárias do beneficiamento (LEAL, 2003). Segundo Banat et al. (1996), quando esses corantes são descartados, sem o devido tratamento, podem permanecer no ambiente por até 50 anos, comprometendo a estabilidade dos ecossistemas aquáticos e elevando os riscos à saúde pública.

Atualmente, existe por volta de 10 mil tipos de corantes diferentes, utilizados principalmente nas indústrias químicas, os quais após aplicações são descartados, em associação com ostros efluentes, ocasionando grandes impactos no meio ambiente. Um desses corantes é o azul de metileno (FREITAG, 2013). Este corante é um composto aromático heterocíclico, sólido, verde escuro, solúvel em água, inodoro, com fórmula molecular $\mathrm{C}_{16} \mathrm{H}_{18} \mathrm{CIN}_{3} \mathrm{~S}$ e massa molar igual a $319,85 \mathrm{~g} / \mathrm{mol}$ (WIKIPEDIA, 2017). Segundo Alfredo (2015), o azul de metileno é um corante catiônico da classe dos fenotiazinas, com diversas aplicações nas áreas da química e cadeia carbônica aromática polinucleada (Figura 1).

Figura 1 - Representação da fórmula estrutural do azul de metileno

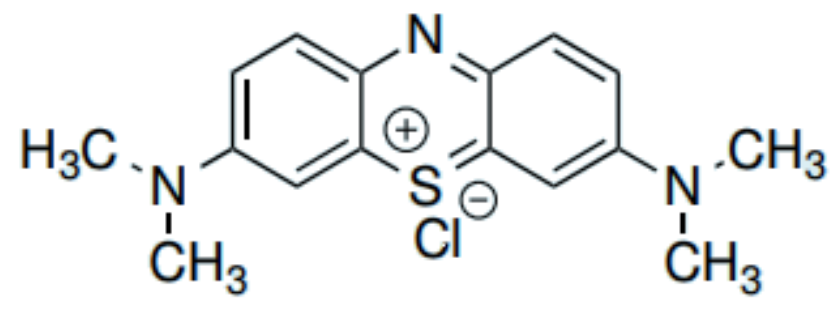

A presença desses corantes no meio ambiente além de causar poluição visual e prejuízos a biota aquática, algumas classes deles podem ser carcinogênicos e/ou mutagênicos. Devido aos inúmeros problemas que podem causar ao ambiente, muitas 
tecnologias têm sido estudadas e desenvolvidas visando a degradação e/ou imobilização desses agentes presentes em efluentes têxteis (RUTZ et al.,2008). Um dos procedimentos capazes de remover o azul de metileno em efluentes aquosos é a adsorção e sua determinação é feita por espectrofotometria UV-VIS, em comprimento de onda máximo de 665 nm (FREITAG, 2013).

A adsorção é uma técnica que tem apresentado resultados satisfatórios na remoção de corantes têxteis em efluentes e também prioridade em relação às outras técnicas devido a sua simplicidade de processamento, seu baixo custo e à variedade de materiais potencialmente utilizáveis. Recentemente, muitas fibras naturais - biomassas - têm sido experimentadas com essa finalidade e, por sua vez, apresentado resultados bastantes promissores. Na literatura estão relacionadas várias biomassas com potencial na remoção de poluentes, seja no estado in natura ou modificada quimicamente, como casca de coco (SOUZA et al., 2007), de arroz (PENHA, 2009), de melancia sugar baby (COSTA; OLIVEIRA, 2017), entre outros. Adsorventes de baixo custo, in natura, também já foram testados e avaliados na remoção da cor de efluentes industriais, tipo cascas de banana e de maracujá (PAVAN et al., 2007), casca de tangerina (FORGACS; CSEHÁTI; OROS, 2004) etc.

Os materiais adsorventes (biomassas) são sólidos que possuem estruturas porosas, que quase sempre apresentam um desequilíbrio de cargas elétricas em suas superfícies, o que proporciona a atração do adsorbato (poluente) presente no fluido. Para caracterizar o quanto essa superfície está eletricamente desestabilizada, determina-se o ponto de carga zero (pHpcz), o qual indicará o valor do pH em que a superfície do material adsorvente (biomassa) apresentará equilíbrio entre as cargas positivas e as cargas negativas, ou seja, carga nula. A importância da determinação desse parâmetro, para a adsorção, é que através dele pode-se prever a melhor faixa de pH na qual o adsorvente será mais efetivo. Neste caso, para valores de $\mathrm{pH}_{\text {solução }}<\mathrm{pH} \mathrm{PCz}$, o material terá um comportamento de cargas superficiais carregadas positivamente, favorecendo a adsorção de ânions; e, para valores de $\mathrm{pH}_{\text {solução }}>$ pHPCz, a superfície do material terá cargas negativas, tendendo a adsorver cátions (NASCIMENTO et al, 2014).

O material escolhido para esse estudo foi o resíduo da casca do limão taiti ( $\mathrm{C} i$ trus Latifolia), que compõe uma biomassa bastante abundante, nas estações de safra 
desse cítrico, e que é totalmente descartado no meio ambiente por falta de aplicabilidade rentável nas comunidades envolvidas na sua produção (MELHOR COM SAÚDE, 2015).

Neste contexto, esta pesquisa teve como objetivo avaliar o ponto de carga zero (pHpcz) e a capacidade de adsorção das fibras da casca do limão taiti, lavadas com água destilada e modificadas quimicamente, na remoção do corante azul de metileno, em sistemas aquosos.

\section{MATERIAL E MÉTODOS}

\subsection{Obtenção do material adsorvente}

As cascas de limão foram inicialmente lavadas em água corrente para limpeza e remoção de resíduos sólidos indesejáveis. Logo após, foram cortadas em fatias com dimensões de aproximadamente 2,0 x 2,0 cm e colocadas de molho em água destilada, a temperatura ambiente $\left(25^{\circ} \mathrm{C}\right)$, por 30 minutos. Em seguida, procedeu-se a secagem do material em estufa com circulação de ar (LUCADEMA), a $60^{\circ} \mathrm{C}$, durante $48 \mathrm{~h}$. As cascas secas foram trituradas em moinho de facas (SOLAB - SLS 35) e tamisadas, obtendo-se um pó com granulometria homogênea inferior a $425 \mathrm{~nm}$. O material obtido foi estocado em refrigerador, a $5{ }^{\circ} \mathrm{C}$, recebendo a denominação de fibra lavada (FL).

\subsection{Modificação química do material adsorvente}

Para realizar a modificação química das fibras de limão taiti adicionou-se $16 \mathrm{~g}$ da fibra lavada $(\mathrm{FL})$ a $800 \mathrm{~mL}$ de uma solução de hidróxido de sódio $(\mathrm{NaOH}) 0,10 \mathrm{M}$, permanecendo sob agitação, à temperatura ambiente, por 2 horas. Em seguida, a parte sólida foi separada da parte líquida por centrifugação (FANEM Exselsa II). Logo após, o material sólido foi lavado, várias vezes, com água destilada até atingir o pH próximo da neutralidade. $\mathrm{O}$ material obtido foi seco em estufa, a $60^{\circ} \mathrm{C}$ durante 48 horas. Ao final dessa operação, o material foi estocado em refrigerador, a $5{ }^{\circ} \mathrm{C}$, recebendo uma nova denominação, fibra modificada (FM). 


\subsection{Preparo das soluções para determinação do pHPCZ}

Na preparação das soluções à diferentes concentrações hidrogeno-iônica, pH variando de 1,0 a 12,0, foram utilizadas água destilada e soluções aquosas de ácido clorídrico $(\mathrm{HCl})$ e de hidróxido de

sódio $(\mathrm{NaOH})$. Com auxílio de um pHmetro de bancada (LUCADEMA) foi medido o $\mathrm{pH}$ das soluções preparadas. $\mathrm{O}$ ajuste da acidez/basicidade das soluções com $\mathrm{pH}<7$ foi feito a partir de diluições de $\mathrm{HCl} \mathrm{0,10} \mathrm{mol} / \mathrm{L}$; e, as de $\mathrm{pH}>7$ a partir de diluições de $\mathrm{NaOH} \mathrm{0,10} \mathrm{mol/L.} \mathrm{As} \mathrm{medidas} \mathrm{do} \mathrm{pH}$ inicial e final foram feitas com as soluções em agitação constante, utilizando um agitador magnético (IKG HS-7).

\subsection{Determinação do ponto de carga zero (pHPcz)}

Para a determinação do pHpcz utilizou-se a técnica dos 11 pontos (REGALBUTO; ROBLES, 2004), com algumas adaptações. O resíduo seco da casca do limão (50 mg), lavado e/ou modificado, foi pesado em balança analítica e colocado em contato com $50 \mathrm{~mL}$ de cada solução aquosas preparada, num erlenmeyer com capacidade de $125 \mathrm{~mL}$, perfazendo um total de 12 soluções a diferentes pH's (1,0 a 12,0), para cada tipo de biomassa obtida. Todos os experimentos foram feitos em triplicata. Após colocação da mistura nos erlenmeyer, estes foram lacrados com papel filme e transferidos para mesa agitadora (SL-180/DT), onde permaneceu, por 24h, sob agitação à $100 \mathrm{rpm}$ e a $25^{\circ} \mathrm{C}$. Após $24 \mathrm{~h}$ de equilíbrio entre as fases sólida-líquida, as soluções foram filtradas e novamente feitas as análises para determinações dos pH's finais. O valor do pHpcz foi calculado pela média dos pontos em que o $\mathrm{pH}$ final foi praticamente constante.

\subsection{Preparação das soluções de azul de metileno}

As soluções foram preparadas diluindo-se uma solução estoque de azul de metileno, $\mathrm{C}_{16} \mathrm{H}_{18} \mathrm{CIN}_{3} \mathrm{~S}$, cuja concentração inicial era de $50 \mathrm{mg} / \mathrm{L}$ e pH próximo da neutralidade $(\mathrm{pH} \approx 7,0)$. Ao final obteve-se 06 (seis) soluções com concentrações de $\mathrm{C}_{16} \mathrm{H}_{18} \mathrm{CIN}{ }_{3} \mathrm{~S}$ iguais a $5,10,20,30,40$ e $50 \mathrm{mg} / \mathrm{L}$. 


\subsection{Procedimento para ensaio da adsorção}

Com auxílio de uma proveta, mediu-se $50 \mathrm{~mL}$ de cada solução preparada e transferiu-se para erlenmeyer de $125 \mathrm{~mL}$ de capacidade, no qual já havia colocado 50 mg das fibras (FL e/ou FM). Os ensaios foram realizados em triplicatas, os erlenmeyers foram postos em mesa agitadora durante 24 horas, a $100 \mathrm{rpm}$ e $25^{\circ} \mathrm{C}$. Logo após, foi realizada a separação, através de filtração simples, e medida a concentração final do azul de metileno nos filtrados por meio de um espectrofotômetro UV/vis (Thermo Scientific Evolution 60S).

A quantidade (q) de azul de metileno adsorvido, em mg/g, e a taxa de adsorção ( $\left.T_{a d}, \%\right)$, das fibras, FL e FM, foram calculadas utilizando-se as Equações 1 e 2, respectivamente.

$$
\begin{gathered}
q=\frac{\left(C_{o}-C_{e q}\right) \cdot V}{m} \\
T_{a d}=\frac{\left(C_{o}-C_{e q}\right)}{C_{o}} \cdot 100
\end{gathered}
$$

onde, $C_{o}$ e $C_{e q}$ correspondem as concentrações de azul de metileno nas soluções inicial e no equilíbrio, em $\mathrm{mg} / \mathrm{L}$, respectivamente; $V$ é o volume da solução aquosa, em L; e $m$ é a quantidade de fibra utilizada, em $\mathrm{g}$.

\subsection{Análise estatística}

Para análise estatística dos resultados foi empregado o programa computacional Sisvar - Versão 5.6. Foram realizadas análises de variância (ANOVA) e teste de Tukey para comparação das médias, com nível de significância de $5 \%(P \leq 0,05)$.

\section{RESULTADOS E DISCUSSÃO}

\subsection{Determinação do ponto de carga zero (PCZ)}

O pH PCz indica o ponto de equilíbrio de cargas na superfície do material adsorvente, o ponto em que o $\mathrm{pH}$ se mantém constante. $\mathrm{O}$ valor do $\mathrm{pH} P C z$ foi determinado pelo cálculo da média dos pontos em que o pH final das soluções permaneceu prati- 
camente constante, assumindo assim um efeito tampão, naquela faixa de $\mathrm{pH}$. Na Figura 2 estão representadas duas situações para a relação entre o pH inicial e $\circ \mathrm{pH}$ final das soluções antes e após imersão do pó adsorvente, respectivamente, após lavagem com água destilada e, em seguida, modificado quimicamente com $\mathrm{NaOH}$ $0,10 \mathrm{M}$.

Figura 2 - Representações para as relações entre os pH inicial e final das soluções antes e após com tato com as fibras lavadas com água destilada e modificada quimicamente, respectivamente

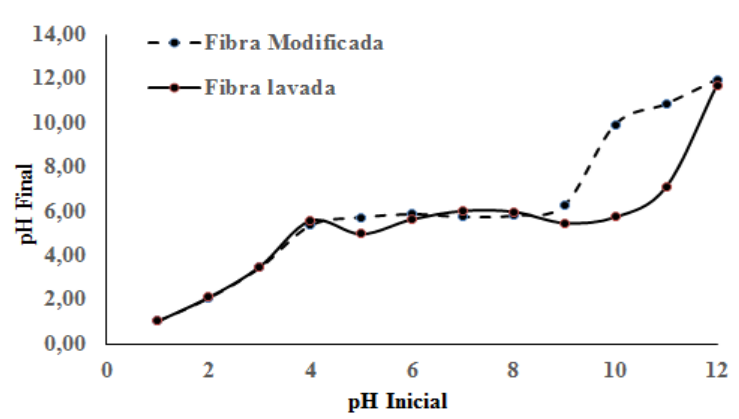

(A)

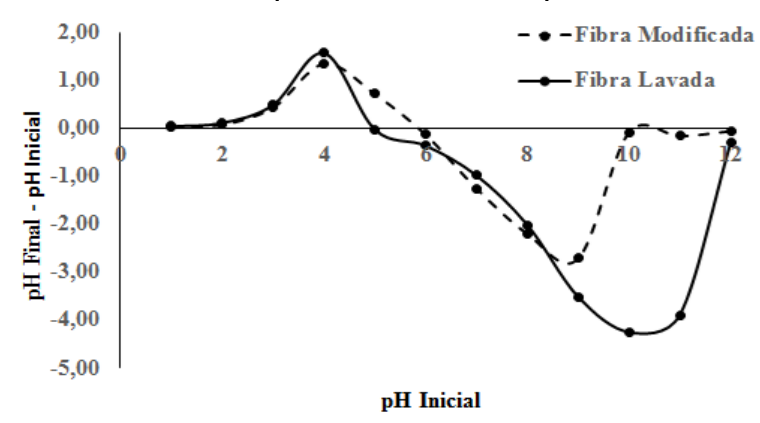

(B)

De acordo com a Figura 2A, é possível verificar que na faixa de $\mathrm{pH}$ inicial $4 \mathrm{a}$ 9, praticamente não há variação para o pH final das soluções experimentadas, após contato por $24 \mathrm{~h}$ com a biomassa da casca do limão taiti. Observa-se, ainda, que esse comportamento foi semelhante para as duas biomassas analisadas. Logo, para a faixa de $\mathrm{pH}$ considerada, os valores dos $\mathrm{pH}_{\mathrm{PCZ}}$ das biomassas testadas foram iguais a 5,63 e 5,80, que para efeito de comprovação, corresponde aos pontos de interseção das respectivas curvas com o eixo das abcissas (Figura $2 \mathrm{~B}$ ), para as fibras lavadas e modificadas, respectivamente, cujos valores não diferenciam entre si $(P \leq 0,05)$, com média igual a 5,72. Este fato indica que os tratamentos aplicados não proporcionaram mudanças no equilíbrio de cargas nas superfícies das cascas do limão taiti. Os resultados foram inferiores ao observado para a casca da banana, que foi de 7,21 (FREITAS; CÂMARA; MARTINS, 2015) e para a casca da laranja, que foi de 6,53 (FORMICA et al., 2017), mas bastante superior ao encontrado para o resíduo do caulim, que foi de 3,73 (PINTO; MAIS; FELIPE, 2012).

Com esta característica, quando a biomassa do limão taiti, seja lavada com água destilada ou modificada quimicamente com $\mathrm{NaOH} \mathrm{0,10} \mathrm{M}$, ao entrar em contato com uma solução aquosa de $\mathrm{pH}$ inferior ao do $\mathrm{pH} P c z$, a superfície é carregada positivamente e ânions serão adsorvidos para balancear as cargas positivas. Por outro lado, se o pH da solução aquosa for superior ao do pHPCZ, a superfície é carregada negativamente e adsorve, preferencialmente, cátions. 


\subsection{Adsorção do azul de metileno}

Embora não exista diferenciação no $\mathrm{pH} p \mathrm{pc}$ das fibras analisadas, observa-se (Tabela 1) que, para todas as concentrações das soluções testadas, a remoção de azul de metileno pelas fibras modificadas foi significativamente maior que pelas fibras lavadas $(P \leq 0,05)$. Isto pode ser devido o azul de metileno ser um corante catiônico e, assim, se ligar mais facilmente à superfície das fibras modificadas, que possuem, ligeiramente, um maior número de grupos com cargas opostas (GUPTA; SUHAS, 2009).

Tabela 1 - Valores médios para a quantidade de azul de metileno adsorvido pelas fibras das cascas do limão taiti $(\mathrm{mg} / \mathrm{g})$, lavadas com água destilada e modificada quimicamente

\begin{tabular}{ccccccc}
\hline Tratamentos & \multicolumn{7}{c}{ Concentração das soluções } \\
\hline & 5 & 10 & 20 & 30 & 40 & 50 \\
\hline Fibra lavada & $4,320 \mathrm{a}$ & $8,504 \mathrm{a}$ & $17,342 \mathrm{a}$ & $26,606 \mathrm{a}$ & $36,595 \mathrm{a}$ & $42,187 \mathrm{a}$ \\
Fibra modificada & $4,977 \mathrm{~b}$ & $9,950 \mathrm{~b}$ & $19,908 \mathrm{~b}$ & $29,816 \mathrm{~b}$ & $39,770 \mathrm{~b}$ & $49,691 \mathrm{~b}$ \\
\hline
\end{tabular}

Média seguidas pela mesma letra nas colunas não diferem entre si pelo Teste de Tukey, ao nível de $5 \%(P \leq 0,05)$.

A taxa de adsorção pelas fibras da casca do limão manteve-se praticamente constante com o aumento da concentração de azul de metileno nas soluções aquosas, tanto para as fibras lavadas quanto modificadas (Figura 3). Ambas biomassas apresentaram taxa de remoção deste corante superior a $80 \%$, sendo as máximas e mínimas iguais a $91,49 \%$ e $84,37 \%$, para a lavada e $99,54 \%$ e $99,38 \%$, para a modificada, respectivamente.

Dessa forma, a adsorção máxima para as fibras ocorreu no contato com as soluções à $50 \mathrm{mg} / \mathrm{L}$, sendo igual a 42,187 mg/g com as lavadas com água destilada e 49,691 mg/g com as modificadas com NaOH. Logo, a taxa de adsorção de azul de metileno na solução aquosa, pelas fibras modificadas, foi superior às obtidas por Gregório et al. (2012) que foi 91\%, com fibra de babaçu e, Furmanski, Costa e Dominguini (2012) que foi de $90 \%$, utilizando a fibra de bananeira modificada. Portanto, as fibras das cascas do limão taiti, modificadas quimicamente com $\mathrm{NaOH}$ 0,10 M é uma alternativa viável para a remoção deste pigmento em sistemas aquosos. 
Figura 3 - Variação da taxa de adsorção (\%) de azul de metileno pelas fibras da casca do limão taiti, lavadas com água destilada (A) e modificadas com $\mathrm{NaOH}$ 0,10 M (B), em função da concentração inicial $\left(\mathrm{C}_{0}\right)$

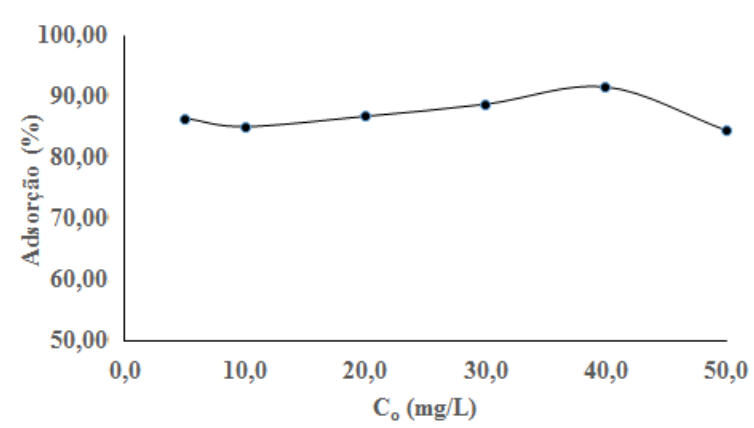

(A)

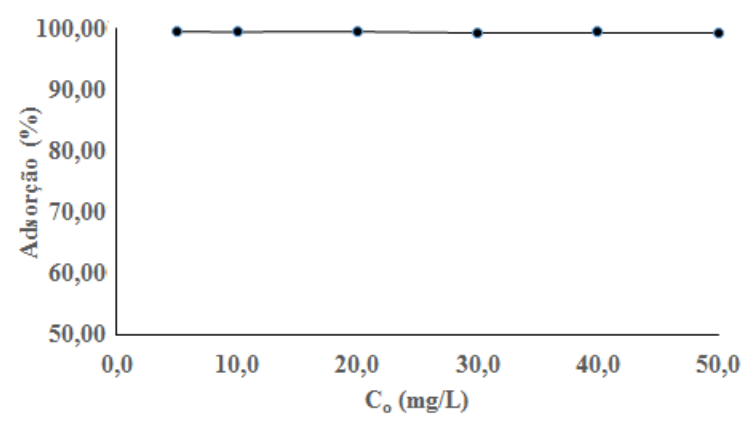

(B)

\subsection{Ajuste ao modelo de adsorção}

Na Tabela 2 encontram-se os parâmetros obtidos pelos ajustes dos dados através dos modelos de Freundlich e Langmuir, das unidades de equilíbrio, para as cascas do limão taiti, nas condições lavada e modificada quimicamente.

Tabela 2 - Parâmetros de ajuste dos modelos das isotermas de adsorção para as cascas do limão taiti, lavadas (FL) e modificadas quimicamente (FM) e coeficiente de determinação $\left(R^{2}\right)$

\begin{tabular}{ccccccc}
\hline Modelo & \multicolumn{2}{c}{ Parâmetros - FL } & \multicolumn{2}{c}{ Parâmetros - FM } & \multicolumn{2}{c}{$\mathbf{R}^{2}$} \\
\hline Langmuir & $q_{\max }$ & $K_{L}$ & $q_{\max }$ & $K_{L}$ & FL & FM \\
& 370,37 & 0,0193 & 172,413 & 1,2608 & 0,0385 & 0,7912 \\
& $1 / \mathrm{n}$ & $K_{F}$ & $1 / \mathrm{n}$ & $K_{F}$ & $\mathrm{FL}$ & $\mathrm{FM}$ \\
\multirow{2}{*}{ Freundlich } & 1,0287 & 6,714 & 0,8834 & 143,119 & 0,9201 & 0,9935 \\
\hline
\end{tabular}

De acordo com as informações existentes na Tabela 2, é possível observar que o processo de adsorção se ajustou melhor a isoterma de Freundlich, cujos coeficientes de determinação $\left(R^{2}\right)$, para as biomassas lavada e modicada, foram iguais a 0,9201 e 0,9935 , respectivamente. Este fato indica que o processo ocorre em multicamadas e como a biomassa modificada apresentou $1<\mathrm{n}<10$, a remoção do azul de metileno foi favorecida nela, em relação a lavada $(n<1)$, indicando que na primeira a interação entre o adsorvato e o adsorvente foi bastante forte, mesmo que possivelmente apresente diferentes energias nos sítios. Sendo o valor de $1 / n>1$, na biomassa lavada, 0 adsorvente tem maior afinidade pelo solvente, sugerindo que há uma forte atração intermolecular entre os dois (DELLE-SITE, 2001). 
$\mathrm{Na}$ Figura 4 tem-se a comparação gráfica dos modelos de Langmuir e Freundlich, por análise de regressão linear, para o azul de metileno adsorvido nas duas biomassas.

Figura 4 - Ajuste dos modelos de Langmuir e Freundlich, por regressão linear, para a adsorção de azul de metileno pelas biomassas, respectivamente, em função da concentração no equilíbrio $\left(\mathrm{C}_{\mathrm{e}}\right)$
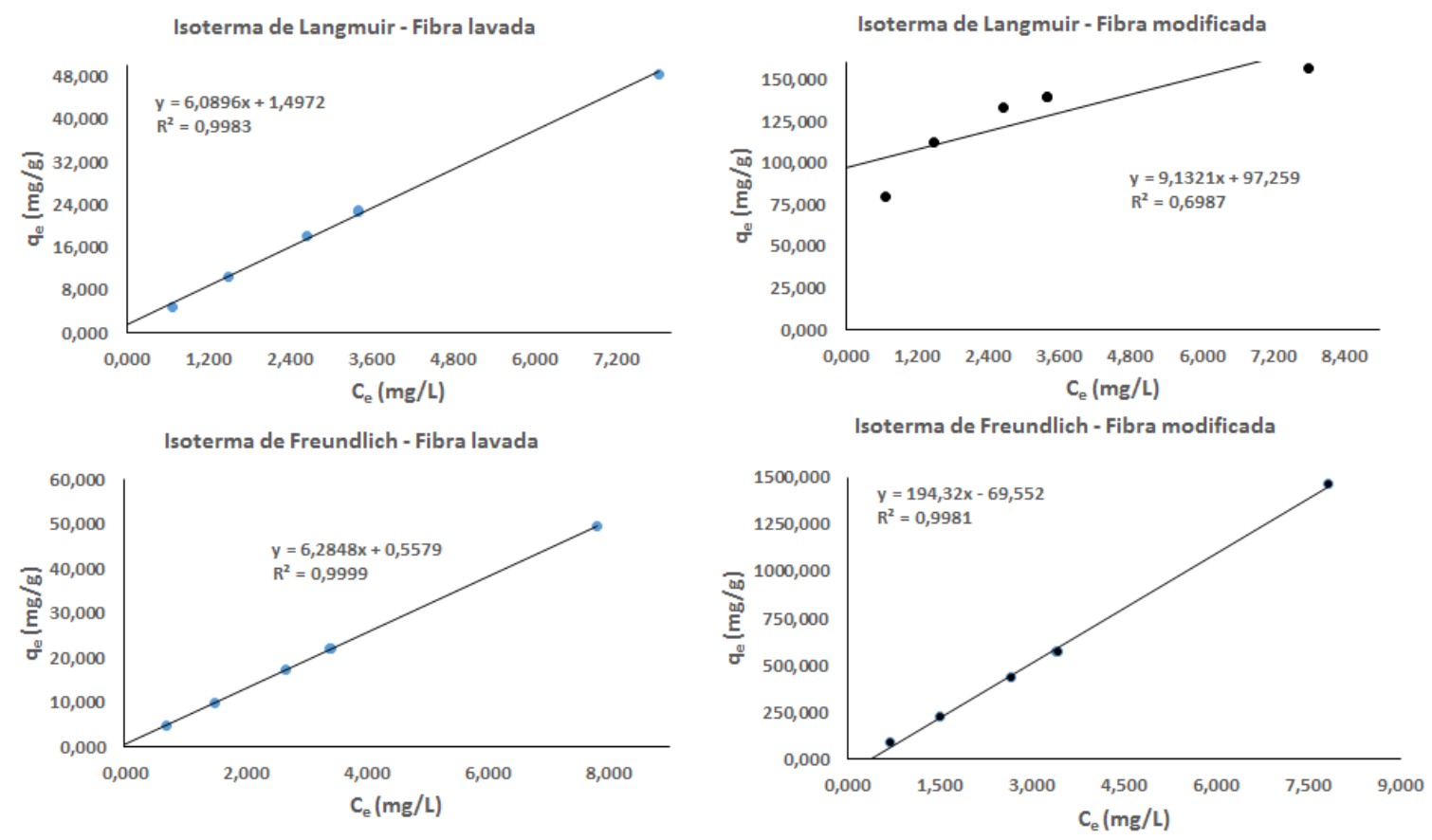

Percebe-se, por meio da Figura 4, o bom ajuste dos dados experimentais ao modelo de adsorção do azul de metileno, pelas cascas do limão taiti, à isoterma de Freundlich. Neste trabalho, a capacidade de adsorção experimental segue a ordem crescente: fibra lavada < fibra modificada.

\section{CONCLUSÕES}

Os tratamentos físicos e químicos aplicados às fibras da casca do limão taiti não modificaram o equilíbrio de cargas elétricas na superfície da biomassa analisada, cujo valor médio do pHpcz foi igual a 5,72. Contudo, a adsorção de azul de metileno pela fibra modificada foi significativamente superior à da fibra lavada com água destilada.

A taxa de adsorção do azul de metileno, pelas duas biomassas, permaneceu praticamente constante com o aumento da concentração das soluções. No entanto, 
na biomassa modificada esse parâmetro manteve-se mais uniforme, com magnitude máxima igual a 99,54\%, correspondendo a uma adsorção de 49,691 mg/g.

A isoterma de adsorção do azul de metileno pelas cascas do limão taiti, lavadas com água destilada e modificadas quimicamente, se ajustou melhor ao modelo de Freundlich, indicando que o processo foi favorável.

\section{AGRADECIMENTOS}

Somos gratos à toda equipe de gestores do Instituto Federal de Educação, Ciência e Tecnologia do Rio Grande do Norte - Campus Nova Cruz pelo apoio material e humana, os quais foram imprescindíveis à conclusão desta pesquisa.

\section{REFERÊNCIAS}

ALFREDO, A. P. C. Adsorção de Azul de Metileno em Casca de Batata Utilizando Sistemas em Batelada e Coluna de Leito Fixo. Rev. Virtual Quim. v. 7, n. 6, p. 1909-1920, 2015.

BANAT, I. M.; NIGAM, P.; SINGH, D.; MARCHANT, R. Microbial decolorization of textile-dyecontaining effluents: a review. Bioresource Technology. v. 58, p.217-227, 1996.

COSTA, D. M. A.; OLIVEIRA, R. A. Adsorção de cromo por resíduos da casca da melancia sugar baby (Citrullus Lanatus). In: Simpósio Nordestino de Química, 3, 2017, Campina Grande. Anais. Campina Grande-PB, 2017.

DELLE-SITE, A. Factors affecting sorption of organic compounds in natural sorbent/water systems and sorption coefficients for selected pollutants. A review. Journal of Physical and Chemical Reference Data, v. 30, p. 187-439, 2001

FORGACS, E.; CSEHÁTI, T.; OROS, G. Removal of synthetic dyes from wastewaters: A review Environment International. v. 30, p. 953-971, 2004.

FORMICA, B. C.; BRUDZINSKI, P. B.; CARVALHO, K. Q.; FLORIANO, J. B.; PASSIG, F. H.; LIZ, M. V. Caracterização e Avaliação das Propriedades Adsortivas da Casca de Laranja na Remoção do Corante Direct Blue 86. Rev. Virtual Quim. v. 9, n. 2, p. 608-625, 2017.

FREITAG, J. Adsorção do corante azul de metileno na rama de mandioca (manihot esculenta crantz). 2013. $41 \mathrm{f}$. Trabalho de Conclusão de Curso (Monografia) - Universidade Tecnológica Federal do Paraná, Toledo, 2013.

FREITAS, F. B. A.; CÂMARA, M. Y. F; MARTINS, D. F. F. Determinação do PCZ de adsorventes naturais utilizados na remoção de contaminantes em soluções aquosas. In: 5 Encontro Regional de Química \& 4 Encontro Nacional de Química, 2015, Mossoró. Blucher Chemistry Proceedings, Mossoró-RN, 2015. 
FURMANSKY, L.; COSTA, P.; DOMINGUINI, L. Estudo da cinética de adsorção de azul de metileno por resíduos fibrosos de bananeira. In: Simpósio Brasileiro de Engenharia Ambiental, 2012, Criciúma. Anais. Criciúma, 2012.

GREGÓRIO, A. M.; SILVA, P. R.; FARIA-KRAUSER, M. O.; LEAL, P. V. B. Influência do pH na adsorção de azul de metileno em Babaçu in natura. In: Congresso Brasileiro de Química, 62, 2012, Recife. Anais. Recife, 2012.

GUPTA, V. K.; SUHAS. Application of low-cost adsorbents for dye removal: a review. J. Environ. Manage, v. 90, n.8, p. 2313 - 2342, 2009.

LEAL, C. C. A. Avaliação da remoção do corante Remazol Black B em efluentes têxteis utilizando com adsorvente mesocarpo de coco verde. 2003. $73 \mathrm{f}$. Dissertação (Mestrado em Engenharia Química). Departamento de Pós-Graduação em Engenharia Química - Universidade Federal de Pernambuco, Recife, 2003.

MELHOR COM SAÚDE - Aproveite os benefícios da casca do limão. Disponível em: $<$ https://melhorcomsaude.com.br/aproveite-o-poder-curativo-do-suco-da-casca-de-limao/>. Acesso em: 06 dez. 2018.

NASCIMENTO, R. F.; LIMA, A. C. A.; VIDAL, C. B.; MELO, D. Q.; RAULINO, G. S. C.. Adsorção: aspectos teóricos e aplicações ambientais. Editora Imprensa Universitária, Ed. Universidade Federal do Ceará. Fortaleza, 2014.

PAVAN, F. A.; GUSHIKEM, Y.; MAZZOCATO, A. S.; DIAS, S. L. P.; LIMA, E. C. Biosorption on yellow passion fruit and mandarin peels. Dyes and Pigments, v. 72, p. 256-266, 2007.

PENHA, R. S. Casca de arroz como adsorvente para íons de metais pesados: caracterização e modificação química. 2009. 88 f. Dissertação (Mestrado). Mestrado em Química Analítica. Universidade Federal do Maranhão. São Luís, 2009.

PINTO, R. L. S.; MAIS, R. F. S.; FELIPE, A. M. P. F. Determinação do PCZ de resíduo do caulim da região do nordeste do Pará. In: Congresso de Cerâmica, 56, Curitiba, Anais. Curitiba, 2012.

REGALBUTO, J. R.; ROBLES, J. The engineering of Pt/Carbon Catalyst Preparation. University of Illinois, Chicago. 2004.

RODRIGUES, K.; VIDAL, C. B.; BARBOSA, B. A. A.; WANDERLEY, C. R. P.; DUARTE, I. C. S.; MARINHO, G. Viabilidade do tratamento de água residuária sintética têxtil em reator aeróbico de leito fixo. Revista Engenharia Sanitária e Ambiental, v. 15, n. 1, p. 99-106, 2010.

RUTZ, E. G.; CARVALHO, C. F.; PIRES, C. S.; CURIEL, G. L. Adsorção de corante têxtil Amarelo de Remazol usando um dos rejeitos da fabricação de alumina como adsorvente. Revista Escola de Minas, v. 61, n. 4, p. 443-448, 2008.

SOUSA, F. W.; Moreira, S. A.; Oliveira, A. G.; Cavalcante, R. M.; Nascimento, R. F.; Rosa, M. F. Uso da casca de coco verde como adsorvente na remoção de metais tóxicos. Química Nova, v. 30, n. 5, p. 1153-1157, 2007.

WIKIPEDIA. 2017. Azul de metileno. Disponível em: https://pt.wikipedia.org/wiki/Azul_de_metileno.

Acesso em: 27 jan. 2018. 